\title{
O efeito de um programa de atividades rítmicas na interação social e na coordenação motora em crianças com transtorno do espectro autista
}

\author{
The effect of a program of rhythmic activities on social interaction and motor \\ coordination in children with autism spectrum disorder
}

\begin{tabular}{l} 
AUTORES \\
\hline Gabriele Radünz Krüger $^{1}$ (D) \\
Luciana Maia Garcias $^{1}$ (D) \\
${\text { Gabriela Padilha Hax }{ }^{1} \text { (D) }}^{\text {Alexandre Carriconde Marques }{ }^{1} \text { (D) }}$ \\
1 Universidade Federal de Pelotas. Pelotas, Rio \\
Grande do Sul, Brasil. \\
CONTATO \\
Gabriele Radünz Krüger \\
gabrielerk@gmail.com \\
Rua Guilherme Leitzke, 171, Três Vendas, \\
Pelotas, Rio Grande do Sul, Brasil. \\
CEP: $96065-684$. \\
DOI
\end{tabular}

$10.12820 /$ rbafs.23e0046

\begin{abstract}
RESUMO
Este estudo tem como objetivo verificar o efeito de um programa de atividades rítmicas na interação social e coordenação motora de crianças com transtorno do espectro autista (TEA). Realizou-se um delineamento experimental com nove crianças com TEA, entre cinco e 10 anos (cinco no grupo intervenção e quatro no grupo controle). Os dados foram obtidos por meio de três instrumentos aplicados antes e após intervenção. Os pais reportaram informações sobre o estilo de vida e interação social das crianças (CARS) e um teste de desenvolvimento motor grosso (TGMD-2) foi aplicado com as crianças. O grupo intervenção realizou 14 semanas, duas sessões por semana de 50 minutos cada, de atividades de dança. O grupo controle foi apenas acompanhado nas atividades diárias. Os dados foram comparados por meio da estatística não-paramétrica, com $\mathrm{p}<0,05$. O grupo intervenção apresentou melhora nas habilidades motoras após as 14 semanas ( $p=0,042$; tamanho do efeito de 1,86). Em relação a interação social não foram observadas melhoras significativas em nenhum dos grupos. Conclui-se que 14 semanas de atividades rítmicas pode ser uma ferramenta eficaz para desenvolver as habilidades motoras de crianças com transtorno do espectro autista.
\end{abstract}

Palavras-chave: Transtorno autístico; Dança; Exercício.

\section{ABSTRACT}

The aim of the present study is to examine the effect of an intervention with rhythmic activities on the motor coordination and social interaction of children with autism spectrum disorder (ASD). The research is an experimental study. The sample consisted of children with ASD between the ages of five and 10 years. The data were obtained through three instruments applied before and after the intervention: two questionnaires, answered by parents or guardians: one about lifestyle and one about social interaction (CARS) and a gross motor development test (TGMD-2) performed with the children. No significant differences were found between groups after 14 weeks of activities. However, only the intervention group presented improvements from the first evaluation for the second evaluation (pre and post) $(p=0,042$; effect size of 1.86) in motor skills. Regarding social interaction, no significant improvements were observed in either group. We conclude that 14 weeks of rhythmic activities can be an interesting tool to develop the motor skills of children with autism spectrum disorder.

Keywords: Autistic Disorder; Dancing; Exercise.

\section{Introdução}

O Transtorno do Espectro do Autismo (TEA) provoca comprometimento em habilidades sociais e de comunicação, nos comportamentos restritos e estereotipados dos indivíduos ${ }^{1}$. Além disso, outra característica marcante são os déficits motores ${ }^{1}$. Observa-se que esses déficits nas habilidades motoras podem prejudicar a participação nas atividades físicas (AF) deste grupo ${ }^{2}$.

Dados internacionais indicam que o percentual de crianças inativas no mundo tem aumentando conside- ravelmente e, nas crianças com TEA, esses números são ainda mais expressivos ${ }^{3}$. Estudos em várias regiões do mundo apontam que as pessoas com TEA apresentam baixos níveis de $\mathrm{AF}^{4-6}$ e esses números se tornam maiores conforme o avanço da idade e o grau de comprometimento do transtorno ${ }^{5}$. Estudos com indivíduos com TEA $^{4-9}$ têm mostrado benefícios da prática de atividade física na melhora na qualidade do sono ${ }^{10}$, na coordenação motora ${ }^{11}$, na melhoria da condição física ${ }^{12}$, entre outros.

Contudo, ainda são poucos os estudos que têm ex- 
plorado o efeito de intervenções que as atividades rítmicas podem trazer para as pessoas com TEA. Kock et al. ${ }^{13}$ realizaram uma intervenção terapêutica de movimentos de dança baseada em espelhamento de movimento com 31 adultos jovens com TEA leve. Após as sete semanas os participantes do grupo de intervenção obtiveram melhora no bem-estar, na consciência corporal, na distinção entre os outros e nas habilidades sociais.

O déficit na interação social é uma das características marcantes das pessoas com TEA, que reflete na dificuldade de relacionamento em diversos ambientes na vida desses indivíduos. A prática de $\mathrm{AF}$ tem se mostrado uma ferramenta eficaz na melhoria das habilidades sociais nas suas diversas formas. $\operatorname{Pan}^{14}$, relatou uma melhora nas habilidades sociais por meio de uma intervenção com atividades de natação em 16 crianças com TEA. Os resultados deste estudo podem direcionar o interesse dos pesquisadores e professores da área, a desenvolverem atividades de dança com crianças com TEA em diversos ambientes, principalmente na escola juntamente com outras crianças sem TEA.

Deste modo, este estudo tem como objetivo verificar o efeito de um programa de atividades rítmicas na interação social e coordenação motora de crianças com transtorno do espectro autista.

\section{Métodos}

O presente estudo trata de um delineamento experimental. A população foi constituída por crianças com TEA, de ambos os sexos, entre cinco e 10 anos, residentes na cidade de Pelotas, Rio Grande do Sul.

A seleção da amostra foi realizada de forma intencional em instituições que atendem crianças com TEA na cidade de Pelotas - Núcleo de Neurodesenvolvimento da Universidade Federal de Pelotas e o Projeto Carinho, desenvolvido pela Escola Superior de Educação Física. Este procedimento de seleção da amostra objetivou a participação do maior número de crianças. De acordo com os pressupostos da pesquisa experimental a divisão dos grupos controle e intervenção foi de forma aleatória, pareando os sujeitos por idades e sexo, sendo assim, ficaram cinco no grupo controle e cinco no grupo intervenção. Foram considerados elegíveis as crianças com diagnóstico médico de TEA, entre cinco e 10 anos de idade, todas com disponibilidade para participar das atividades duas vezes na semana. $\mathrm{O}$ tamanho da amostra apresentou um poder maior de 0,80 para as comparações realizadas entre os grupos nos desfechos analisados (análise realizada após a cole- ta de dados pelo programa Gpower 3.1).

O grupo intervenção realizou um programa de 14 semanas, com duas sessões semanais de 50 minutos de dança. Os conteúdos das aulas trabalharam a coordenação motora ampla, ritmo, equilíbrio e socialização. As aulas eram dividas em três partes, a primeira era composta por uma roda inicial, na qual, todos deveriam sentar-se em um círculo e os monitores relatavam o que seria realizado na aula; a parte principal consistia em um aquecimento com atividades cantadas e após era realizada atividades com músicas variadas - com as músicas eram realizadas pequenas coreografias propostas pelos alunos e pelos monitores, além disso eram realizadas danças já conhecidas como a da cantora Xuxa (cabeça, ombro, joelho e pé), muitas vezes eram realizadas cantadas, eram feitas também brincadeiras como, por exemplo: quem é o chefe, a dança da cadeira comunitária, entre outras; e na parte final era feito um relaxamento com alongamento. O grupo controle não recebeu nenhum tipo de tratamento, apenas realizou os testes de avaliação.

Os indivíduos dos dois grupos foram avaliados em dois momentos, pré e pós-intervenção. Foram avaliados quanto o estilo de vida, a interação social e coordenação motora de crianças. $\mathrm{O}$ estilo de vida foi avaliado por questionário ${ }^{15}$, respondido pelos pais ou responsáveis. Este questionário foi adaptado para características da população com TEA (visto que o original tratava de pessoas com Síndrome de Down) e analisou os dados de identificação e familiar (dados gerais das crianças, informações educacionais e características sócio demográficas) e característica do estilo de vida (questões relacionadas a $\mathrm{AF}$, atividades de vida diária, atividades habituais nos momentos livres).

As interações sociais foram avaliadas pela escala "Childhood Autism Rating Scale (CARS)", ou Escala de Avaliação do Autismo na Infância ${ }^{16}$ o qual foi traduzido e validado para utilização no Brasil por Pereira ${ }^{17}$, o questionário foi respondido pelos pais ou responsáveis, em razão da dificuldade de entendimento das crianças com TEA. A escala é composta por 15 itens que abrange questões acerca das relações pessoais, uso do corpo, dos sentidos e de objetos, resposta a mudanças, comunicação verbal e não verbal e consistência cognitiva, que auxilia na identificação de crianças com TEA e as distingue de crianças com prejuízos no desenvolvimento. É breve, e seu uso é apropriado para qualquer criança acima de dois anos de idade $^{16}$. Cada item possui uma escala de sete pontos, que variam de um a quatro sendo que possuem valores intermediários 
(1-1, 5-2-2, 5-3-3, 5-4). Os resultados definem a seguinte pontuação: $15-30$ : sem autismo; 30-36: autismo leve-moderado; 36-60: autismo grave ${ }^{16}$.

O "Test of Gross Motor Development" (TGMD$2)^{18}$, o qual foi traduzido e validado para utilização no Brasil por Valentini ${ }^{19}$, avalia o desenvolvimento motor amplo em habilidades de locomoção e controle de objetos, foi utilizado para avaliar a coordenação motora. Neste estudo, apenas foram testadas as habilidades de locomoção, composta por sete itens (corrida, galopar, saltitar, salto sobre o mesmo pé, salto com o ambos os pés e corrida lateral). $O$ protocolo do teste sugere a utilização de uma câmara filmadora para gravar e após analisar o desempenho motor. Cada sub item possui vários critérios de desempenho de acordo com análise do vídeo: a criança recebia um (1) ponto se conseguisse realizar de maneira correta o critério e nenhum ponto se ele não fosse realizado. O TGMD também foi aplicado antes e depois da intervenção.

Para análise dos dados foram utilizados recursos de estatística não paramétrica, onde foram observadas as medidas de tendência central (médias e desvio padrão) e frequências, para a descrição dos resultados. Para verificar a diferença entre os grupos foi utilizado o teste Mann Whitney e para avaliar as diferenças intragrupos nos momentos pré e pós-intervenção foi utilizado o teste Wilcoxon. Além disso foi calculado o tamanho do efeito estandardizado mediante o d de Cohen ${ }^{20}$. O programa SPSS versão 20.0 foi utilizado na análise dos dados.

O estudo foi submetido e aprovado sobre o número de protocolo 035/2012, pelo Comitê de Ética em Pesquisa com Seres Humanos da Escola Superior de Educação Física da Universidade Federal de Pelotas (ESEF/UFPel). Todos os envolvidos tiveram o Termo de Consentimento Livre e Esclarecido assinado, concordando com a participação, mantendo os preceitos éticos de pesquisas com seres humanos.

\section{Resultados}

Participaram do estudo 10 crianças entre cinco e 10 anos, sendo cinco no grupo controle e cinco no grupo intervenção). Ao final do estudo um participante do grupo controle foi excluído por não ter realizado o segundo teste, gerando assim uma perda.

Os grupos controle e intervenção apresentaram composição semelhantes em relação as características sociodemográficas (Tabela 1). Contudo, os participantes do grupo intervenção participavam em maior número de escolas comuns, de aulas de Educação Físi- ca e atividade física no lazer. Outras características da amostra está apresentada na Tabela 1.

Tabela 1 - Características da amostra de crianças com Transtorno de Espectro de Autismo, Pelotas, Rio Grande do Sul, 2012 ( $\mathrm{n}=9$ ).

\begin{tabular}{|c|c|c|}
\hline $\begin{array}{l}\text { Variáveis/ } \\
\text { Categorias }\end{array}$ & $\begin{array}{c}\text { Grupo intervenção } \\
(\mathrm{n}=5)\end{array}$ & $\begin{array}{c}\text { Grupo controle } \\
(\mathrm{n}=4)\end{array}$ \\
\hline \multicolumn{3}{|l|}{ TEA } \\
\hline Leve a moderado & 2 & 1 \\
\hline Severo & 3 & 3 \\
\hline \multicolumn{3}{|l|}{ Sexo } \\
\hline Meninos & 4 & 3 \\
\hline Meninas & 1 & 1 \\
\hline \multicolumn{3}{|l|}{ Idade (anos) } \\
\hline 6 & 1 & 1 \\
\hline 7 & 2 & 2 \\
\hline 9 e 10 & 2 & 1 \\
\hline \multicolumn{3}{|l|}{ Cor da pele } \\
\hline Branca & 4 & 3 \\
\hline Não-branca & 1 & 1 \\
\hline \multicolumn{3}{|c|}{ Presença de alguma doença } \\
\hline Sim & 3 & 2 \\
\hline Não & 2 & 2 \\
\hline \multicolumn{3}{|c|}{ Frequente escola comum } \\
\hline Sim & 4 & 1 \\
\hline Não & 1 & 3 \\
\hline \multicolumn{3}{|c|}{ Participa das aulas EDF } \\
\hline Sim & 3 & 1 \\
\hline Não & 2 & 3 \\
\hline \multicolumn{3}{|l|}{ Faz $\mathrm{AF}^{\mathrm{b}}$ no lazer } \\
\hline Sim & 4 & 2 \\
\hline Não & 1 & 2 \\
\hline
\end{tabular}

$\mathrm{a}=$ Educação Física; $\mathrm{b}=$ Atividade Física.

Em relação as habilidades motoras observa-se que através do teste Mann Whitney os grupos diferiram no início da intervenção $(\mathrm{p}=0,017)$ e mantiveram essa diferença após o termino da intervenção $(\mathrm{p}=0,013)$ - Tabela 2. Além disso, observou-se que houve uma melhora significativa das habilidades motoras no grupo intervenção $(p=0,042)$, com um tamanho do efeito de 1,86 ( $\mathrm{d}$ de Cohen). O grupo controle não apresentou diferença significativa $(\mathrm{p}=0,317)$ entre os momentos. O tamanho do efeito entre os grupos foi de $\mathrm{d}=2,04$.

No que concerne a interação social, no momento pré-teste os grupos eram semelhantes $(\mathrm{p}=0,556)$, e se mantiveram após as 14 semanas de intervenção ( $\mathrm{p}$ $=0,111)-$ Tabela 2 . Ambos os grupos não apresentaram diferenças significativas da pré e pós-intervenção. O grupo experimental apresentando uma $d=-1,35$ e o grupo controle $d=0,52$. Ao comparar o tamanho do efeito entre ambos os grupos verificamos uma $\mathrm{d}=-1,69$. 
Tabela 2 - Efeito da intervenção baseada em exercícios físicos nas habilidades motoras e interação social de crianças com Transtorno de Espectro de Autismo, Pelotas, Rio Grande do Sul, 2012 ( $\mathrm{n}=9$ ).

\begin{tabular}{|c|c|c|c|c|c|c|c|c|c|c|c|c|c|}
\hline \multirow{2}{*}{$\begin{array}{l}\text { Variável/ } \\
\text { Momento }\end{array}$} & \multicolumn{6}{|c|}{ Grupo intervenção $(\mathrm{n}=5)$} & \multicolumn{6}{|c|}{ Grupo controle $(n=4)$} & \multirow{2}{*}{$\begin{array}{c}\text { Valor }-\mathrm{p} \\
\text { entre Grupos }\end{array}$} \\
\hline & Mín & Máx & $\mathrm{P} 25$ & $\mathrm{P} 50$ & P75 & Valor-p & Mín & Máx & $\mathrm{P} 25$ & P50 & $\mathrm{P} 75$ & Valor-p & \\
\hline Habilidades motoras & & & & & & $0,042 \dagger a$ & & & & & & $0,317 \dagger$ & \\
\hline Pré-teste & 6,0 & 23,0 & 8,5 & 12,0 & 19,5 & & 0,0 & 6,0 & 0,0 & 0,0 & 4,5 & & $0,017^{* *}$ \\
\hline Pós-teste & 18,0 & 34,0 & 19,0 & 28,0 & 31,0 & & 0,0 & 6,0 & 0,0 & 2,5 & 5,7 & & $0,013^{* *}$ \\
\hline Interação social & & & & & & $0,060 \dagger$ & & & & & & $0,100 \dagger$ & \\
\hline Pré-teste & 1,5 & 3,0 & 2,0 & 2,5 & 3,0 & & 1,5 & 3,5 & 1,6 & 2,0 & 3,1 & & $0,556^{* *}$ \\
\hline Pós-teste & 1,0 & 2,5 & 1,2 & 1,5 & 2,2 & & 1,5 & 4,0 & 1,7 & 2,7 & 3,7 & & $0,111^{\text {** }}$ \\
\hline
\end{tabular}

* = Uma diminuição na interação social significa uma melhora nesta variável; ** = Teste Mann Whitney; † = Teste Wilcoxon; a = Representa uma diferença significativa entre o momento pré-teste e o momento pós-teste para o grupo.

\section{Discussão}

Os principais achados do presente estudo foram que o grupo intervenção apresentou melhora significativa nas habilidades motoras após 14 semanas de atividades de dança, enquanto o grupo controle manteve os valores dessa variável. Além disso, com relação a interação social ambos os grupos (intervenção e controle) mantiveram os valores após o tratamento.

A partir do que foi observado as atividades rítmicas contribuíram para melhorias nas habilidades motoras de locomoção, conforme resultado dos testes do grupo intervenção. Através destes resultados pode-se concordar com Castro \& Morais $^{21}$, que afirmam que através da dança podemos trabalhar a coordenação motora de forma eficaz ${ }^{21}$. Esses resultados podem auxiliar aos professores Educação Física (EF) na montagem de seus planos de aulas. O efeito da intervenção demonstrou que a utilização de atividades rítmicas promovem a melhora das habilidades motoras. Essas atividades são de baixo custo e podem ser utilizadas nas aulas de EF na escola, servindo como ferramenta na melhoria do processo inclusivo e nas condições de saúde desses indivíduos.

Ao analisar os resultados da interação social, não foi observada melhora significativa, porém observou um tamanho de efeito considerável. Contudo, são diversos os estudos que apontam benefícios da dança nesse quesito. Campeiz \& Volp ${ }^{22}$ relatam que as atividades rítmicas podem melhorar o desenvolvimento social através do jogo imaginativo e das atividades cooperativas, gerando um ambiente amigável e agradável, facilitando assim o aprendizado ${ }^{22}$. Kock et.al. ${ }^{13}$ realizaram uma intervenção terapêutica de movimentos de dança baseada em espelhamento de movimento com 31 adultos jovens com TEA leve e identificaram melhorias nas habilidades sociais ${ }^{13}$.

Em relação a intervenção, acredita-se que os participantes obtiveram melhorias devido as atividades rítmicas, as quais eram bem estruturadas, sendo objetivo de cada aula trabalhar duas habilidades motoras e a interação social. Todavia, este tipo de atividade ainda é muito pouco explorada, ou pelo menos relatada no campo da pesquisa e pode trazer um efeito positivo a esta população.

Os resultados encontrados no presente estudo são relevantes, porém deve-se levar em conta a existência da limitação da amostra selecionada ser reduzida impossibilitando a generalização dos resultados à população. Contudo observa-se que o efeito das atividades rítmicas nas habilidades motoras de crianças com TEA foi positivo, mostrando que essas atividades podem ser uma excelente ferramenta no desenvolvimento de crianças com TEA.

Tendo ciência das limitações motoras dos indivíduos com TEA ${ }^{23}$ notou-se uma melhora em tal condição. Neste sentido pode-se supor que as atividades rítmicas propiciam uma melhora nas habilidades motoras avaliadas (corrida, corrida lateral, passada, galope, salto horizontal e salto monopedal), podendo auxiliar na melhoria da qualidade de vida destes indivíduos.

A falta de estudos de intervenção na área envolvendo pessoas com TEA é relevante, entretanto, observou-se que as atividades rítmicas são um conteúdo interessante a ser trabalhado nessa população, e desta forma acredita-se que os resultados encontrados possam auxiliar na aquisição de novos conhecimentos e subsidiar novas pesquisas sobre o tema. $\mathrm{O}$ efeito desta intervenção nas habilidades motoras mostrou-se relevante, e estimular a prática destas atividades em vários ambientes poderá trazer benefícios na mudança do estilo de vida dessas crianças e seus familiares. Diante do apresentado, novas discussões e novas propostas de intervenções para beneficiar essa parcela da população poderão ser desenvolvidas em novas pesquisas. Além disso o avanço do diagnóstico de crianças com TEA tem aumentado a prevalência desses casos, o que de certa forma pode se apresentar como um alerta para o desenvolvimento de novas pesquisas e 
a melhora do atendimento dessa população em diversos ambientes, principalmente na escola.

Diante dos resultados encontrados, conclui-se que um programa de atividades rítmicas, com duração de 14 semanas, melhorou as habilidades motoras os participantes. Mesmo não apresentando uma diferença significativa, percebe-se uma tendência positiva na melhoria da interação social.

\section{Conflito de interesses}

Os autores declaram não haver conflito de interesses.

\section{Financiamento}

A pesquisa foi realizada com recursos dos próprios pesquisadores.

\section{Agradecimentos}

Os autores agradecem as famílias e as crianças com TEA que se dispuseram a participar de um estudo longo como esse, oportunizando a melhora do conhecimento na área, que poderá estimular a outros pesquisadores na busca para novos desafios.

\section{Contribuição dos autores}

Krüger GR, participou das etapas de concepção do artigo, coleta de dados, intervenção, análise e interpretação de dados, escrita e revisão final do texto. Garcias LM, participou da coleta de dados, tabulação dos dados e revisão final do texto. Hax GP, participou da coleta de dados, escrita e revisão final do texto. Marques AC, participou das etapas de concepção do artigo, coleta de dados, intervenção, análise e interpretação de dados, escrita e revisão final do texto.

\section{Referências}

1. American Psychiatric Association. Diagnostic and statistical manual of mental disorders-DSM-V. 5th ed. Washington: Artmed, 2013.

2. Stins JF, Emck C. Balance Performance in Autism: A Brief Overview. Front Psychol. 2018;9:901.

3. Krüger GR. Atividade física e barreiras em crianças com autismo de Pelotas [dissertação de mestrado]. Pelotas, Rio Grande do Sul: Universidade Federal de Pelotas; 2015.

4. Macdonald M, Esposito P, Ulrich D. The physical activity patterns of children with autism. BMC research notes. 2011;4:422.

5. Memari AH, Ghaheri B, Ziaee V, Kordi R, Hafizi S, Moshayedi P. Physical activity in children and adolescents with autism assessed by triaxial accelerometry. Pediatr Obes. 2013;8(2):150-58.
6. Pan CY, Frey GC. Physical activity patterns in youth with autism spectrum disorders. J Autism Dev Disord.2006;36(5):597-606.

7. Memari AH, Panahi N, Ranjbar E, Moshayedi P, Shafiei M, Kordi, et al. Children with Autism Spectrum Disorder and Patterns of Participation in Daily Physical and Play Activities. Neurol Res Int. 2015;2015:531906-7.

8. Pan CY. Objectively measured physical activity between children with autism spectrum disorders and children without disabilities during inclusive recess settings in Taiwan. J Autism Dev Disord. Aug 2008;38(7):1292-1301.

9. Pan CY, Tsai CL, Chu CH, Sung MC, Ma WY, Huang CY. Objectively Measured Physical Activity and HealthRelated Physical Fitness in Secondary School-Aged Male Students With Autism Spectrum Disorders. Phys Ther. 2016;96(4):511-20.

10. Brand S, Jossen, S, Trachsler E, Pühse U, Gerber M. Impact of aerobic exercise on sleep and motor skills in children with autism spectrum disorders - a pilot study. Neuropsychiatr Dis Treat. 2015;11:1911-20.

11. Macdonald M, Lord C, Ulrich D A. Motor skills and calibrated autism severity in young children with autism spectrum disorder. Adapt Phys Activ Q. 2014;31:95-105.

12. Pitetti KH, Rendoff AD, Grover T, Beets MW. The efficacy of a 9-month treadmill walking program on the exercise capacity and weight reduction for adolescents with severe autism. J Autism Dev Disord, 2007;37:997-1006.

13. Koch SC, Mehl L, Sobanski E, Sieber M, Fuchs T. Fixing the mirrors: A feasibility study of the effects of dance movement therapy on young adults with autism spectrum disorder. Autism. 2015;19(3):338-50

14. Pan CY. Effects of water exercise swimming program on aquatic skills and social behaviors in children with autism spectrum disorders. Autism. 2010; 14(1):9-28.

15. Marques AC. O perfil do estilo de vida de pessoas com Síndrome de Down e normas para avaliação da aptidão física [tese de doutorado]. Porto Alegre, Rio Grande Sul: Educação física Universidade Federal do Rio Grande do Sul; 2008.

16. Schopler ERR, Renner BR. The Childhood Autism Rating Scale (CARS). 10. ed. Los Angeles, CA: Western Psychological Services, 1988.

17. Perreira AM. Autismo infantil: Tradução e Validação da CARS (Childhood Autism Rating Scale) para uso no Brasil [dissertação de Mestrado]. Porto Alegre, Rio Grande do Sul: Universidade Federal do Rio Grande do Sul; 2007.

18. Ulrich D. The test of gross motor development. Austin: ProdEd; 2000.

19. Valentini N. Percepções de Competência e Desenvolvimento Motor de meninos e meninas: um estudo transversal. Revista Movimento. 2002; 8(2):51-62.

20. Cohen J. (1988). Statistical power analysis for the behavioral sciences (2nd ed.). Hillsdale, NJ: Lawrence Earlbaum Associates.

21. Castro ME, Morais R. A influência da dança na percepção de estruturas rítmicas monotônicas em adolescentes surdos. Revista Motricidade. 2013; 9;1:69-86.

22. Campeiz ECFS, Volp MC. Dança criativa: a qualidade da experiência subjetiva. Revista Motriz. 2004;10:167-72.

23. McPhillips M, Finlay J, Bejerot S, Hanley M. Motor Deficits in Children With Autism Spectrum Disorder: A CrossSyndrome Study. Autism Research. 2014;7:664-76.

Recebido: 08/01/2018

Aprovado: 20/01/2019 